\title{
THE PROBLEMS ASSOCIATED WITH FLAIL FEET IN CHILDREN AND THEIR TREATMENT WITH ORTHOSES
}

\author{
G. E. FULFORD, T. P. CAIRNS \\ From the Princess Margaret Rose Orthopaedic Hospital, Fairmilehead, Edinburgh
}

\begin{abstract}
Children with flail feet who have control of their hips and knees are able to walk but are unable to stand still and find it difficult to walk slowly. These problems have been overcome in fifty-three children with flail feet due to myelomeningocele by fitting them with below-knee orthoses which provide maximum stability and yet allow normal walking. The biomechanical principles and the development of the orthoses are discussed.
\end{abstract}

More children with myelomeningocele are now surviving, particularly those with involvement of the lower lumbar spinal cord who have reasonable voluntary control of their hips and knees but whose ankles and feet are either flail or show dorsiflexion but no useful plantarflexion. This distal pattern of paralysis causes difficulty with balance both when standing and when walking. It is usually treated by short calipers with posterior stops or square ferrules (Menelaus 1971; Sharrard 1971) which can be reinforced by blocked leather slip-on below-knee bootees inserted into ordinary shoes (Rose 1976). We have found that these traditional orthoses do not give the necessary stability. This paper considers the biomechanical requirements of these children to enable independent standing and walking, and describes the development of a suitable orthosis.

\section{BIOMECHANICS OF THE ANKLE DURING STANDING AND WALKING}

The centre of gravity of the body lies just in front of the second segment of the sacrum, and if the body is not to topple while attempting to stand still, its line of action must fall within the area of support provided by the feet. Because the forefoot is longer than the hindfoot the line of action of the centre of gravity can move further forward than backward relative to the ankle, before it falls outside the supporting base (Fig. 1). An extreme example of this is seen in the circus clown who, because his feet are strapped into very large rigid-soled shoes, is able to lean far forward without toppling over (Fig. 2).

Because the ankle joint allows movement between the body and the supporting base, the system is stabilised by muscle action. Normally a person stands with the line of action of the centre of gravity just in front of the axis of the ankle joint so that there is always a tendency for the body to fall forwards. It does not do so because of the strong plantarflexors acting on the forefoot lever which, as the foot is stationary on the ground, pull the leg backwards and restore it to the near vertical position (Fig. 3).

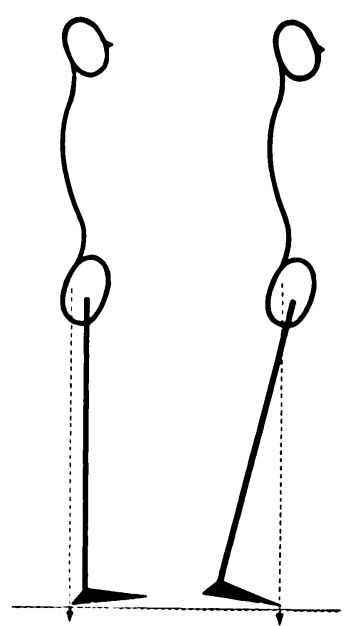

Fig. 1

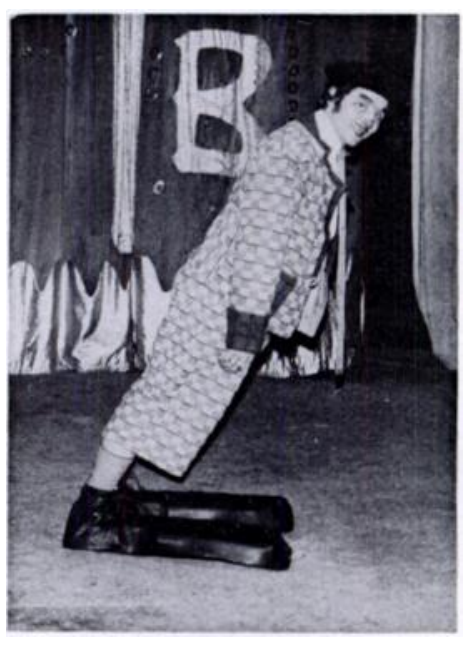

Fig. 2
Figure 1-Diagrams to show that the line of action of the centre of gravity can move further forward than backward before toppling. Figure 2-A circus clown showing the stability obtained from rigid-soled boots.

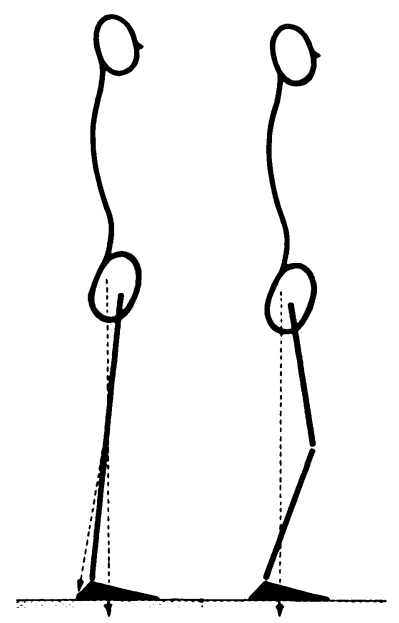

Fig. 3

Normally the forward moment of the centre of gravity in front of the ankle joint is balanced by the action of the plantarflexors behind the ankle joint. When the plantarflexors are paralysed the forward moment of the centre of gravity causes a forward movement of the tibia until it is resisted by the ligaments of the ankle joint in full dorsiflexion. 
During walking the line of action of the centre of gravity moves from behind the ankle at heel-strike to in front of the ankle at mid-stance. From mid-stance onwards the plantarflexors act, in the same way as they do during standing, to allow controlled dorsiflexion and thus stabilise the body over the foot while the other leg swings through. At the end of the stance phase a smooth roll onto the forefoot is allowed by dorsiflexion of the toes. During the swing phase the forefoot is kept clear of the ground by the action of dorsiflexors which continue to control plantarflexion at heel-strike, so that the foot does not slap down on the ground.

Patients with paralysis of the plantarflexors of both feet have lost the normal means of stabilising the body over the feet. When standing, this instability has the same effect as when a normal person stands on the points of his heels: they are unable to stand still because their feet must continuously chase after the line of action of their centre of gravity to prevent a fall. This is very tiring. One way of overcoming this instability is to allow children alter their speed of walking by altering the length of their stride rather than its cadence. This can be seen when they try to walk slowly for they take small rapid steps; they are unable to stroll. A few children prefer the stability gained by walking with hips and knees flexed, and the ankles dorsiflexed. Lack of dorsiflexion causes a drop-foot gait during the swing phase.

\section{DEVELOPMENT OF THE NEW ORTHOSIS}

An orthosis is required which stabilises the ankle during standing so that the foot can be used as a supporting base with the leg in the vertical position. The usual way to achieve this is with a below-knee caliper using double uprights with flat spurs fitting into a flat socket in the heel of the shoe. The uprights must be aligned slightly forwards so that the centre of gravity is in front of the ankle joint and the resulting tendency to fall forwards is resisted by the forefoot lever. With this system it was found that the sole of the shoe soon softened and distorted into a position of dorsiflexion so that the patient returned to the crouched position. If this was overcome by stiffening the shoe with a sole-plate from the heel socket to the front of the toe, it was necessary to provide a rocker-bottom sole to preserve a smooth roll onto the front of the

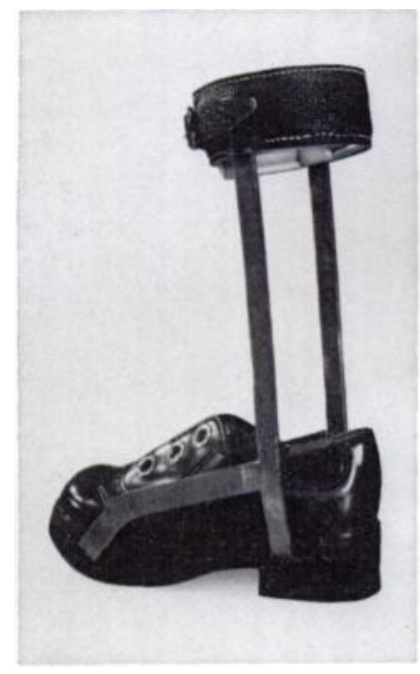

Fig. 4

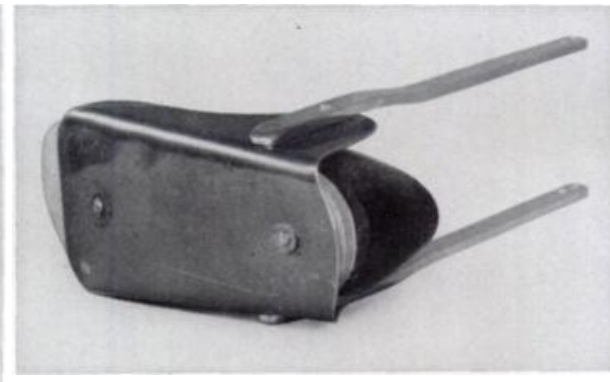

Fig. 5

Figure 4-A standard below-knee orthosis with flat spurs and sole plate strengthened by struts. Figure 5-Aluminium alloy sole-plate and uprights riveted to the base of the shoe. Figure 6The orthosis ready for alignment fitting.
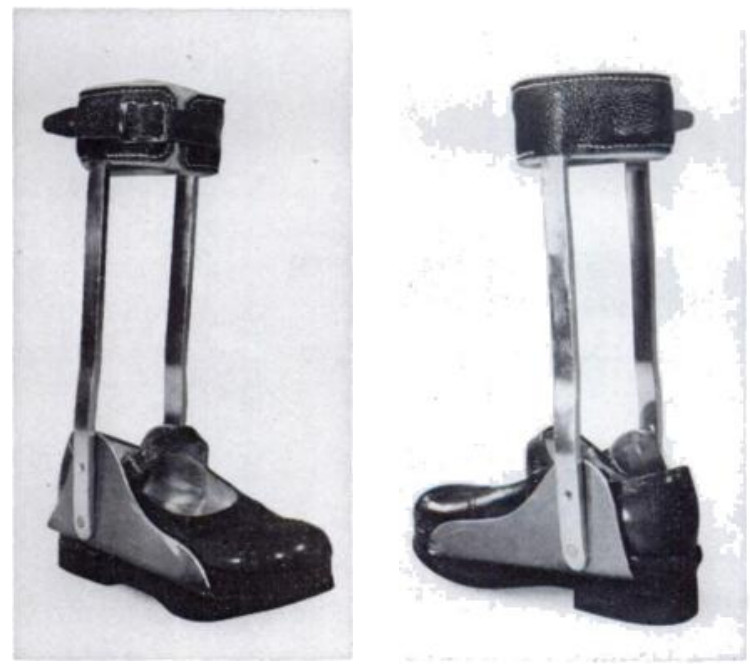

Fig. 6 the foot to move into full dorsiflexion so that, when the feet are on the ground, the legs are leaning forwards. In order to bring the line of action of the centre of gravity back over the feet, the hips and knees must be flexed and the patient stands in a crouched position (Fig. 3). This is a stable position but also tiring because of the increased power required to stabilise the hips and knees in the flexed position (Perry, Antonelli and Ford 1975). Alternatively, the child may achieve stability with the hips and knees straight by using a stick or by holding onto a stable object.

When walking the effect of this instability is less obvious. The inability to plantarflex during the stance phase is unimportant providing the other limb swings through quickly enough to allow heel-strike before the line of action of the centre of gravity has fallen too far in front of the supporting limb. This means that these shoe when walking. This modified caliper at first gave much more stability but soon failed, particularly in the active child, when either the spurs bent or the socket broke away from the shoe. This problem was overcome by placing a strut between the front of the sole-plate and the upright of the side iron (Fig. 4). The orthosis then worked satisfactorily but was heavy, unsightly and complicated to make.

Present orthosis. The orthosis used at present is made from aluminium alloy: the sole-plate is a sheet 2 millimetres thick and the uprights are bars 16 by 5 millimetres. The sole-plate, bent at right angles along the inner and outer aspects of the shoe provides a light and very rigid sole to which the shoe is riveted (Fig. 5). This plate extends from the heel to a point distal to the metatarsal heads, thus preventing any bending of the shoe. The uprights are attached to the sole-plate with 4 millimetre steel rivets on either side of the shoe in the line of the axis of the ankle joint. The uprights are joined at their upper ends by a spring steel calf band and a lined leather adjustable strap across the front of the shin. Lightweight polyethylene foam (P.O. 62) is bonded to the sole-plate and tapered off under the toes. The shoe is soled and heeled with microcellular rubber (Fig. 6).

The correct alignment of the uprights is obtained by fixing them to 
the sole-plate with the lower rivet only. The orthosis is then fitted to the patient and the uprights adjusted until the child can stand vertical without the feeling of falling backwards. The second rivet is then inserted to fix the uprights to the sole-plates in this position.

\section{CLINICAL EXPERIENCE}

We have used this orthosis for the past two years and have fitted fifty-three patients. At first we fitted a single outside plate and upright as this made the sole of the shoe sufficiently rigid. We soon found that with the bigger children and with those who are very active the orthosis broke due to torsion stresses at the junction of the upright either with the calf band or with the soleplate. We have continued to use a single outside upright for small children who are just beginning to walk because weight is an important factor at this stage. In all older children we now fit double uprights. They must be correctly aligned with the shoe so that the line of action of the centre of gravity is in front of the ankle joint but not too far forward. When the uprights have been fixed to the sole-plate by the second rivet the centre of gravity can be brought further forward over the foot by loosening the anterior strap of the calf band, thus increasing the feeling of stability. If the centre of gravity is too far forward this tendency can be checked by putting blocks under the forefoot. Small permanent adjustments can be made by bending the uprights. The correct alignment of the uprights and the shape of the rocker sole are critical if the caliper is to function correctly. Both can be lost when the shoes are repaired unless the repairs are done by someone who understands the principle of the caliper. The rocker must be kept well forward in order to keep the forefoot lever as long as possible but must be tapered to allow a smooth roll over the front of the shoe. We have found that many of the older children have a valgus hindfoot which may be sufficient to allow the inner side of the ankle to come into contact with the inner plate. This can be overcome by cranking the inner vertical part of the sole-plate.

Usually the patient's first comment after the orthoses are fitted is that he is now able to stand still, while the parents often remark that their child appears
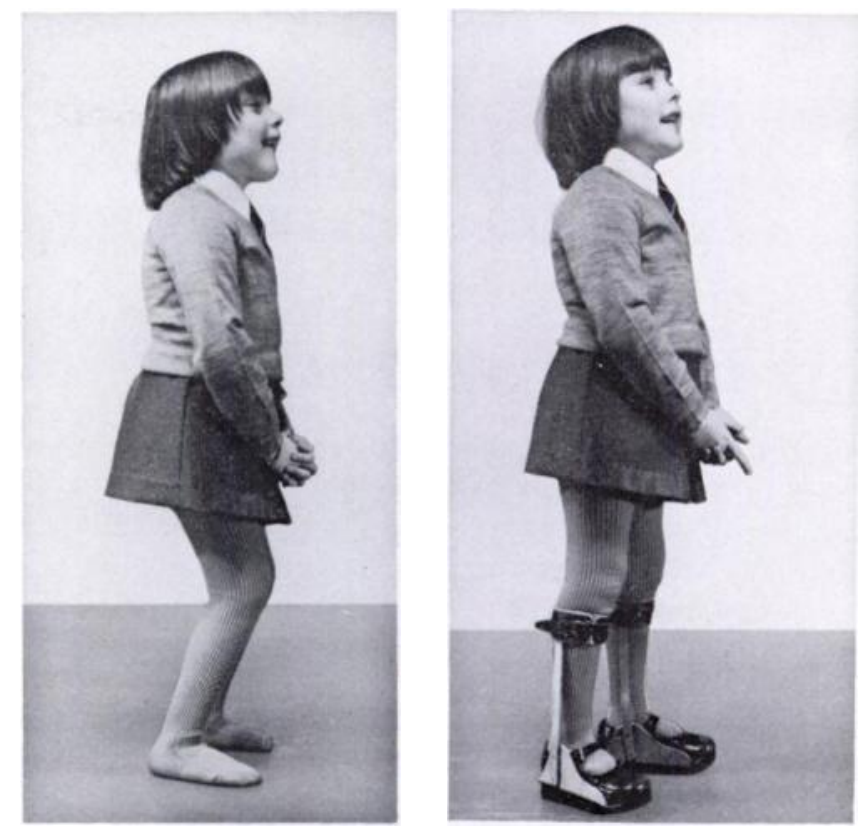

Fig. 7

A child with flail feet gaining stability by fully dorsiflexing the ankles which causes her to stand with flexed knees and hips. Orthoses allow her to stand up straight.

to have grown because he no longer stands in the crouched position (Fig. 7). Later, the child may say he can walk further or that he does not find walking so tiring. The younger child is often able to walk without sticks. In the older children, however, the use of sticks may be desirable to disguise an associated abductor weakness which becomes more apparent as they grow. Grundy et al. (1975) have shown that both increasing the rigidity of the sole of the shoe and the use of a metatarsal bar reduces the forefoot impulse (force-time) compared with the heel impulse. Their orthosis consists of a very rigid sole-plate with an efficient metatarsal bar: by reducing the pressures under the forefoot there is less likelihood of pressure ulcers developing under the metatarsal heads. We have fitted one patient with this orthosis who previously had been troubled by such an ulcer and so far there has been no recurrence.

\section{REFERENCES}

Grundy, M., Tosh, P. A., McLeish, R. D., and Smidt, L. (1975) An investigation of the centres of pressure under the foot while walking. Journal of Bone and Joint Surgery, 57-B, 98-103.

Menelaus, M. B. (1971) The Orthopaedic Management of Spina Bifida Cystica. Edinburgh and London: Churchill Livingstone.

Perry, J., Antonelli, D., and Ford, W. (1975) Analysis of knee-joint forces during flexed-knee stance. Journal of Bone and Joint Surgery, 57-A, $961-967$.

Rose, G. K. (1976) Surgical orthotic management of spina bifida. In The Advance in Orthotics, pp 405-413. Edited by G. Murdoch. London: Edward Arnold.

Sharrard, W. J. W. (1971) Paediatric Orthopaedics and Fractures. Oxford: Blackwell Scientific Publications. 